\title{
Research Article \\ Strict Efficiency in Vector Optimization with Nearly Convexlike Set-Valued Maps
}

\author{
Xiaohong Hu, ${ }^{1}$ Zhimiao Fang, $^{2}$ and Yunxuan Xiong ${ }^{3}$ \\ ${ }^{1}$ Department of Mathematics and Physics, Chongqing University of Posts and Telecommunications, Chongqing 400065, China \\ ${ }^{2}$ Chongqing Police College, Chongqing 401331, China \\ ${ }^{3}$ Basic Course Department, Nanchang Institute of Science and Technology, Nanchang 330108, China
}

Correspondence should be addressed to Xiaohong Hu; huxh@cqupt.edu.cn

Received 10 December 2012; Revised 6 March 2013; Accepted 11 March 2013

Academic Editor: Sheng-Jie Li

Copyright (C) 2013 Xiaohong Hu et al. This is an open access article distributed under the Creative Commons Attribution License, which permits unrestricted use, distribution, and reproduction in any medium, provided the original work is properly cited.

\begin{abstract}
The concept of the well posedness for a special scalar problem is linked with strictly efficient solutions of vector optimization problem involving nearly convexlike set-valued maps. Two scalarization theorems and two Lagrange multiplier theorems for strict efficiency in vector optimization involving nearly convexlike set-valued maps are established. A dual is proposed and duality results are obtained in terms of strictly efficient solutions. A new type of saddle point, called strict saddle point, of an appropriate set-valued Lagrange map is introduced and is used to characterize strict efficiency.
\end{abstract}

\section{Introduction}

One important problem in vector optimization is to find the efficient points of a set. As observed by Kuhn, Tucker, and later by Geoffrion, some efficient points exhibit certain abnormal properties. To eliminate such abnormal efficient points, various concepts of proper efficiency have been introduced. The original concept was introduced by Kuhn and Tucker [1] and Geoffrion [2] and was later modified and formulated in a more general framework by Borwein [3], Hartley [4], Benson [5], Henig [6], and Borwein and Zhuang [7]; also see the references therein. In particular, the concept of strict efficiency was first introduced by Bednarczak and Song [8] in order to obtain upper semicontinuity of the section mapping $G(y)=S \cap(y-C)$ at an efficient point. Zaffaroni [9] used a special scalar function to characterize the strict efficiency and obtained some properties of strict efficiency, which includes well posedness.

Recently, several authors have turned their interests to vector optimization of set-valued maps. For instance, see [10-16]. Li [17] extended the concept of Benson proper efficiency to set-valued maps and presented two scalarization theorems and Lagrange multiplier theorems for set-valued vector optimization problem under cone subconvexlikeness.
Mehra [18] and Xia and Qiu [19] discussed the super efficiency in vector optimization problem involving nearly coneconvexlike set-valued maps and nearly cone-subconvexlike set-valued maps, respectively. Miglierina [20] linked the properly efficient solutions of set-valued vector optimization with well-posedness hypothesis of a special scalar problem.

In this paper, inspired by $[8,17,18]$, we study strict efficiency for vector optimization problem involving nearly cone-convexlike set-valued maps in the framework of real normed locally convex spaces. The paper is organized as follows. In Section 2, we recall some basic concepts and lemmas. In Section 3, the well posedness of a special scalar problems on strict efficiency involving nearly cone-convexlike setvalued maps is discussed. In Section 4, two scalarization theorems for strict efficiency in vector optimization problems involving nearly cone-convexlike set-valued maps are obtained. In Section 5, we establish two Lagrange multiplier theorems which show that strictly efficient solution of the constrained vector optimization problem is equivalent to strictly efficient solution of an appropriate unconstrained vector optimization problem. In Section 6, some results on strict duality are given. In Section 7, a new concept of strict saddle point for set-valued Lagrangian map is introduced and is then utilized to characterize strict efficiency. 


\section{Preliminaries}

Throughout this paper, let $X$ be a linear space and $Y$ and $Z$ two real normed locally convex spaces, with topological dual spaces $Y^{*}$ and $Z^{*}$. For a set $A \subset Y, \operatorname{cl} A$, int $A, \partial A$, and $A^{c}$ denote the closure, the interior, the boundary, and the complement of $A$, respectively. Moreover, we will denote by $B$ the closed unit ball of $Y$. A set $C \subset Y$ is said to be a cone if $\lambda c \in C$ for any $c \in C$ and $\lambda \geq 0$. A cone $C$ is said to be convex if $C+C \subset C$, and it is said to be pointed if $C \cap(-C)=\{0\}$. The generated cone of $C$ is defined by

$$
\text { cone } C:=\{\lambda c \mid \lambda \geq 0, c \in C\} .
$$

The dual cone of $C$ is defined as

$$
C^{+}:=\left\{\varphi \in Y^{*} \mid \varphi(c) \geq 0, \forall c \in C\right\} .
$$

The quasi-interior of $C^{+}$is the set

$$
C^{+i}:=\left\{\varphi \in Y^{*} \mid \varphi(c)>0, \forall c \in C \backslash\left\{0_{Y}\right\}\right\} .
$$

Recall that a base of a cone $C$ is a convex subset $\Theta$ of $C$ such that

$$
0_{Y} \notin \operatorname{cl} \Theta, \quad C=\text { cone } \Theta .
$$

Of course, $C$ is pointed whenever $C$ has a base. Furthermore, if $C$ is a nonempty closed convex pointed cone in $Y$, then $C^{+i} \neq \emptyset$ if and only if $C$ has a base.

Definition 1. Let $S$ be a nonempty subset of $Y$. The set of all strictly efficient points and the set of all efficient points with respect to the convex cone $C$ with nonempty interior are defined as

$$
\begin{gathered}
\operatorname{StE}(S, C):=\{y \mid \text { for every } \epsilon>0, \exists \delta>0 \\
\text { such that }(S-y) \cap(\delta B-C) \subseteq \epsilon B\}, \\
E(S, C):=\left\{y \mid(S-y) \cap-C=0_{Y}\right\} .
\end{gathered}
$$

It is easy to verify that

$$
\operatorname{StE}(S, C) \subseteq E(S, C) \text {. }
$$

Also, in this paper, we assume that $C \subset Y$ and $D \subset Z$ are pointed closed convex cone with nonempty interior. $F$ : $X \rightarrow 2^{Y}$ and $G: X \rightarrow 2^{Z}$ are set-valued maps with nonempty value. Let $L(Z, Y)$ be the space of continuous linear operations from $Z$ to $Y$, and let

$$
L_{+}(Z, Y):=\{T \in L(Z, Y) \mid T(D) \subset C\} .
$$

Let $(F, G)$ be the set-valued map from $X$ to $Y \times Z$, denoted by

$$
(F, G)(x)=F(x) \times G(x) .
$$

If $\varphi \in Y^{*}, T \in L(Z, Y)$, we also define $\varphi F: X \rightarrow 2^{\mathbb{R}}$ and $F+T G: X \rightarrow 2^{Y}$ by $(\varphi F)(x)=\varphi[F(x)]$ and $(F+T G)(x)=$ $F(x)+T[G(x)]$, respectively.
Definition 2 (see [12]). A set-valued map $F: X \rightarrow 2^{Y}$ is said to be nearly $C$-convexlike on $X$ if $\operatorname{cl}(F(x)+C)$ is convex in $Y$.

Lemma 3 (see [12]). Let $F: X \rightarrow 2^{Y}$ be nearly $C$ convexlike set-valued map on $X$. Then exactly one of the following statement holds:

(i) $\exists x \in X$ such that $F(x) \cap(-\operatorname{int} C) \neq \emptyset$,

(ii) $\exists \varphi \in C^{+} \backslash\left\{0_{Y}\right\}$ such that $(\varphi F)(x) \subset \mathbb{R}_{+}$.

Lemma 4 (see $[12])$. If $(F, G)$ is nearly $C \times D$-convexlike on $X$, then

(i) for each $\varphi \in C^{+} \backslash\left\{0_{Y}\right\},(\varphi F, G)$ is nearly $\mathbb{R}_{+} \times D$ convexlike on $X$;

(ii) for each $T \in L_{+}(Z, Y), F+T G$ is nearly $C$-convexlike on $X$.

Lemma 5 (see [21]). Let $S$ be a closed convex subset of $Y$ and $K$ a closed convex pointed cone. Then $S \cap\left(-K \backslash\left\{0_{Y}\right\}\right)=\emptyset$, if and only if there exists $\varphi \in K^{+i}$ such that $\varphi(s) \geq 0$, for all $s \in S$.

\section{Strict Efficiency and Well Posedness}

Consider the following vector optimization problem with setvalued maps:

$$
\begin{aligned}
& C-\min F(x) \\
& \text { s.t. } G(x) \cap(-D) \neq \emptyset, \\
& \qquad x \in X .
\end{aligned}
$$

Denote the feasible solution set of (VP) by

$$
A:=\{x \in X: G(x) \cap(-D) \neq \emptyset\},
$$

And denote the image of $A$ under $F$ by

$$
F(A)=\bigcup_{x \in A} F(x) \text {. }
$$

Definition 6. A point $\bar{x}$ is said to be a strictly efficient solution of (VP), if there exists $\bar{y} \in F(\bar{x})$ such that $\bar{y} \in \operatorname{St} E[F(A), C]$, and the point $(\bar{x}, \bar{y})$ is said to be a strictly efficient minimizer of (VP).

Definition 7. For a set $S \subseteq Y$, let the function $\Delta_{S}: Y \rightarrow$ $\mathbb{R} \cup\{ \pm \infty\}$ be defined as

$$
\Delta_{S}(y)=d_{S}(y)-d_{Y \backslash S}(y),
$$

where $d_{S}(y)=\inf \{\|s-y\|: s \in S\}$ with $d_{\emptyset}(y)=+\infty$.

The function $\Delta$ was first introduced in [22], and its main properties are gathered together in the following proposition.

Proposition 8 (see [9]). If the set $S$ is nonempty and $S \neq Y$, then

(1) $\Delta_{S}$ is real valued;

(2) $\Delta_{S}$ is 1-Lipschitzian; 
(3) $\Delta_{S}(y)<0$ for every $y \in \operatorname{int} A, \Delta_{S}(y)=0$ for every $y \in \partial A$, and $\Delta_{S}(y)>0$ for every $y \in \operatorname{int} S^{c}$;

(4) if $S$ is closed, then it holds that $S=\left\{y: \Delta_{S}(y) \leq 0\right\}$;

(5) if $S$ is a convex, then $\Delta_{S}$ is convex;

(6) if $S$ is a cone, then $\Delta_{S}$ is positively homogeneous;

(7) if $S$ is a closed convex cone, then $\Delta_{S}$ is nonincreasing with respect to the ordering relation induced on $Y$.

We consider the following parameterized scalar problem:

$$
\begin{aligned}
& \min \Delta_{-C}(y-\bar{y}) \\
& \text { s.t. } y \in F(A) .
\end{aligned}
$$

The following theorem characterize the relation between strictly efficient points of (VP) and the parameterized scalar problem $\left(\mathrm{P}_{\bar{y}}\right)$.

Theorem 9. Let $\bar{x} \in A, \bar{y} \in F(\bar{x})$. Then $(\bar{x}, \bar{y})$ is a strictly efficient minimizer of $(V P)$ if and only if there exists a nondecreasing function $\phi: \mathbb{R}_{+} \rightarrow \mathbb{R}_{+}$with $\phi(0)=0$ and $\phi(t)>0$ for all $t>0$, such that $\Delta_{-C}(y-\bar{y}) \geq \phi(\|y-\bar{y}\|)$ for all $y \in F(A)$.

Proof. Since $(\bar{x}, \bar{y})$ is a strictly efficient minimizer of vector optimization problem (VP) can be rephrased as follows: for every $\epsilon>0$ there exists $\delta>0$ such that $d_{-C}(y-\bar{y}) \geq \delta$ for every $y \in F(A)$ with $\|y-\bar{y}\|>\epsilon$. So suppose that the point $(\bar{x}, \bar{y})$ is a strictly efficient minimizer of (VP) and consider the following functions:

$$
\begin{gathered}
\phi_{0}(\epsilon)=\inf \left\{d_{-C}(y-\bar{y}) \mid y \in F(A),\|y-\bar{y}\| \geq \epsilon\right\}, \\
\phi(\epsilon)=\min \left(\phi_{0}(\epsilon), 1\right) .
\end{gathered}
$$

It is evident that $\phi$ is nondecreasing, null at the origin, and positive elsewhere; moreover, for every $y \in F(A)$ it holds that

$$
\Delta_{-C}(y-\bar{y})=d_{-C}(y-\bar{y}) \geq \phi(\|y-\bar{y}\|) .
$$

If, on the other hand, there exists a nondecreasing function $\phi$ with the above properties and such that $\Delta_{-C}(y-\bar{y}) \geq \phi(\| y-$ $\bar{y} \|)$ for all $y \in F(A)$, then it holds that $\Delta_{-C}(y-\bar{y})=d_{-C}(y-$ $\bar{y})>0$ for all $y \in F(A)$ with $y \neq \bar{y}$. To show that $(\bar{x}, \bar{y})$ is a strictly efficient minimizer of (VP), for every $\epsilon>0$, we can let $\delta=\inf \left\{d_{-C}(y-\bar{y}):\|y-\bar{y}\|>\epsilon\right\}$, and it implies that the proof is completed.

The scalar problem $\left(\mathrm{P}_{\bar{y}}\right)$ is Tikhonov well posed if $\Delta_{-C}(y-\bar{y})>0$ for all $y \in F(A)$ with $y \neq \bar{y}$ and

$$
y_{n} \in F(A), \quad d_{-C}\left(y_{n}-\bar{y}\right) \longrightarrow 0 \Longrightarrow y_{n} \longrightarrow \bar{y} .
$$

Theorem 10. Let $\bar{x} \in A, \bar{y} \in F(\bar{x})$. The $(\bar{x}, \bar{y})$ is a strictly efficient minimizer of (VP) if and only if $\bar{y}$ is a solution of $\left(P_{\bar{y}}\right)$ and the scalar problem $\left(P_{\bar{y}}\right)$ is Tikhonov well posed.

Proof. If $(\bar{x}, \bar{y})$ is a strictly efficient minimizer of (VP), then, by Theorem $9, \bar{y}$ is the unique solution of $\left(\mathrm{P}_{\bar{y}}\right)$ and there exists a forcing function $\phi$ such that $\Delta_{-C}(y-\bar{y}) \geq \phi(\|y-\bar{y}\|)$, for all $y \in F(A)$. Since $\phi\left(t_{n}\right) \rightarrow 0$ implies $t_{n} \rightarrow 0$, hence for any sequence $y_{n} \in F(A)$ such that $\Delta_{-C}\left(y_{n}-\bar{y}\right) \rightarrow 0$, then it must converge to $\bar{y}$.

Conversely, if the scalar problem $\left(\mathrm{P}_{\bar{y}}\right)$ is Tikhonov well posed, then $d_{-C}(y-\bar{y})=\Delta_{-C}(y-\bar{y})$ holds for every $y \in F(A)$. Thus, we consider the function $\phi(\epsilon)=\inf \left\{d_{-C}(y-\bar{y}): \| y-\right.$ $\bar{y} \| \geq \epsilon\}$; it holds by the construction that $d_{-C}(y-\bar{y} \geq \phi(y-\bar{y})$, and it is to see that $\phi$ is nondecreasing on $[0, \infty)$ with $\phi(0)=0$ and $\phi(t)>0$ for all $t>0$. Hence, again, by the Theorem 9 , we get that $(\bar{x}, \bar{y})$ is a strictly efficient minimizer of (VP).

\section{Strict Efficiency and Linear Scalarization}

In association with the vector optimization problem (VP) involving set-valued maps, we consider the following linearly scalar optimization problem with a set-valued map:

$$
\begin{aligned}
& \min (\varphi F)(x) \\
& \text { s.t. } x \in A,
\end{aligned}
$$

where $\varphi \in Y^{*} \backslash\left\{0_{Y^{*}}\right\}$.

Definition 11. If $\bar{x} \in A, \bar{y} \in F(A)$ and

$$
\varphi(\bar{y}) \leq \varphi(y), \quad \forall y \in F(A),
$$

then $\bar{x}$ and $(\bar{x}, \bar{y})$ are called a minimal solution and a minimizer of $\left(\operatorname{LSP}_{\varphi}\right)$, respectively.

Lemma 12. Let $\bar{y} \in S \subset Y$, and $C$ is a closed convex cone with having a compact base $\Theta$. Then $\bar{y}$ is a strictly efficient point of $S$ if and only if $\mathrm{cl}(S+C-\bar{y}) \cap-C=\left\{0_{Y}\right\}$.

Proof. The definition of strict efficiency of $S$ can be rephrased as follows: for every $\epsilon>0$, there exists $\delta>0$ such that $d_{-C}(y-$ $\bar{y})>\delta$ for every $y \in S$ with $\|y-\bar{y}\|>\epsilon$. Hence, if there exists sequence $y_{n} \in S, c_{n} \in C$, and some $c \in$ int $C$ such that $y_{n}+c_{n}-\bar{y} \rightarrow-c$, then $y_{n}+c_{n}+c-\bar{y} \rightarrow 0$; hence, $d_{-C}(y-\bar{y}) \rightarrow 0$. Since $y_{n}-\bar{y}=-\left(c_{n}+c\right), c \neq 0$, and $C$ is pointed, $y_{n}-\bar{y}$ is outside some small ball around the origin. This shows that $\bar{y}$ is not the strictly efficient point of $S$, this contraction shows that $\mathrm{cl}(S+C-\bar{y}) \cap-C=\left\{0_{Y}\right\}$.

Conversely, if $\bar{y}$ is not a strictly efficient point of $S$, then there exists $\epsilon>0$ and a sequence $y_{n} \in S$, and $c_{n} \in C$ such that

$$
\left\|y_{n}-\bar{y}\right\| \geq \epsilon, \quad\left\|y_{n}+c_{n}-\bar{y}\right\| \longrightarrow 0 .
$$

We write $c_{n}=\lambda_{n} \theta_{n}$ with $\lambda_{n}>0$ and $\theta_{n} \in \Theta$, then, by (16) and as $\Theta$ is compact, there exists $\alpha>0$ and $N \in R^{+}$such that $\alpha<\lambda_{n}$. Indeed, by (16), we have $c_{n} \notin(\epsilon / 2) B$; furthermore, since $\Theta$ is compact, thus $\lambda_{n}$ does not converse to 0 , and it implies that there exists a real number $\alpha>0$ and $N \in \mathbb{R}^{+}$ such that $\alpha<\lambda_{n}$ for all $n \geq N$. Now, we define $c_{n}^{\prime}=\left(\lambda_{n}-\alpha\right) \theta_{n}$, $n \geq N$.

Thus, we obtain

$$
y_{n}+c_{n}^{\prime}-\bar{y}=y_{n}+c_{n}-\bar{y}-\alpha \theta_{n} \longrightarrow-\alpha \theta \neq 0 .
$$


Hence,

$$
\operatorname{cl}(S+C-\bar{y}) \cap-C \backslash\left\{0_{Y}\right\} \neq \emptyset .
$$

This contradiction shows that $\bar{y}$ is a strictly efficient point of $S$.

Theorem 13. Let $\bar{x} \in A, \bar{y} \in F(\bar{x})$, let $C$ have a compact base, and let $\varphi \in C^{+i}$ be fixed. If $(\bar{x}, \bar{y})$ is a minimizer of $\left(\operatorname{LSP}_{\varphi}\right)$, then $(\bar{x}, \bar{y})$ is a strictly efficient minimizer of $(V P)$.

Proof. By Lemma 12, we need only to prove that

$$
\operatorname{cl}(F(A)+C-\bar{y}) \cap-C=\left\{0_{Y}\right\} .
$$

Indeed, let $c \in \operatorname{cl}(S+C-\bar{y}) \cap-C$. Then there exists $\left\{y_{n}\right\} \subset$ $F(A),\left\{c_{n}\right\} \subset C$ such that

$$
c=\lim _{n \rightarrow+\infty}\left(y_{n}+c_{n}-\bar{y}\right),
$$

hence,

$$
\varphi(c)=\lim _{n \rightarrow+\infty}\left[\varphi\left(y_{n}\right)+\varphi\left(c_{n}\right)-\varphi(\bar{y})\right] .
$$

Since $(\bar{x}, \bar{y})$ is a minimizer of $\left(\operatorname{LSP}_{\varphi}\right)$ and $y_{n} \in F(A)$, we have $\varphi\left(y_{n}\right) \geq \varphi(\bar{y})$, while $c_{n} \in C$ and $\varphi \in C^{+i}$ imply that $\varphi\left(c_{n}\right) \geq 0$. Hence, $\varphi(c) \geq 0$. On the other hand, since $c \in-C$, we have $\varphi(c) \leq 0$. Thus $\varphi(c)=0$. Again, by $\varphi \in C^{+}$and $c \in-C$, we must have $c=0_{Y}$.

Hence, we have shown that $\operatorname{cl}(S+C-\bar{y}) \cap-C=\left\{0_{Y}\right\}$. Therefore, this proof is completed.

Theorem 14. Let $F$ be nearly $C$-convexlike on $A, \bar{x} \in A$, and $\bar{y} \in F(\bar{x})$, and let $C$ have a compact base. If $(\bar{x}, \bar{y})$ is a strictly efficient minimizer of $(V P)$, then there exists $\varphi \in C^{+}$such that $(\bar{x}, \bar{y})$ is a minimizer of $\left(\operatorname{LSP}_{\varphi}\right)$.

Proof. Since $(\bar{x}, \bar{y})$ is a strictly efficient minimizer of (VP), thus by Lemma 12, we have

$$
-C \cap \mathrm{cl}[F(A)+C-\bar{y}]=\left\{0_{Y}\right\} .
$$

By the definition of nearly $C$-convexlike set-valued map $F$, we have that $\mathrm{cl}[F(A)+C-\bar{y}]$ is closed convex set in $Y$, since $C$ is a closed, convex, pointed, compact cone. Thus, by Lemma 5, there exists $\varphi \in C^{+i}$ such that

$$
\varphi(\operatorname{cl}[F(A)+C-\bar{y}]) \geq 0 .
$$

Since

$$
F(A)-\bar{y} \subset F(A)+C-\bar{y} \subset \operatorname{cl}[F(A)+C-\bar{y}],
$$

we obtain

$$
\varphi(y)-\varphi(\bar{y}) \geq 0, \quad \forall y \in F(A) .
$$

Therefore, $(\bar{x}, \bar{y})$ is a minimizer of $\left(\operatorname{LSP}_{\varphi}\right)$.

If we denote by $S t E(\mathrm{VP})$ the set of strictly efficient minimizer of (VP) and by $M\left(\operatorname{LSP}_{\varphi}\right)$ the set of minimizer of $\left(\operatorname{LSP}_{\varphi}\right)$, then from Theorems 13 and 14, we get immediately the following corollary.
Corollary 15. Let F be nearly C-convexlike on X. Then,

$$
\operatorname{StE}(V P)=\bigcup_{\varphi \in C^{+i}} M\left(L S P_{\varphi}\right) .
$$

\section{Strict Efficiency and Lagrange Multipliers}

In this section, we establish two Lagrange multiplier theorems which show that the set of strictly efficient minimizer of the constrained set-valued vector optimization problem (VP), it is equivalent to the set of an appropriate unconstrained vector optimization problem.

The following concept is a generalization of Slater constraint qualification in mathematical programming and in vector optimization.

Definition 16. We say that (VP) satisfies the generalized Slater constraint qualification if there exists $\widetilde{x} \in X$ such that $G(\widetilde{x}) \cap$ $(-\operatorname{int} D) \neq \emptyset$.

Theorem 17. Let $F$ be nearly $C$-convexlike on $X$. Let $(F, G)$ be nearly $C \times D$-convexlike on $X$ and let $C$ have a compact base. Furthermore, let $(V P)$ satisfy the generalized Slater constraint qualification. If $(\bar{x}, \bar{y})$ is a strictly efficient minimizer of $(V P)$, then there exists $T \in L_{+}(Z, Y)$ such that $T[G(\bar{x}) \cap(-D)]=$ $0_{Y}$ and $(\bar{x}, \bar{y})$ is a strictly efficient minimizer of the following unconstrained vector optimization problem:

$$
\begin{aligned}
& C-\min F(x)+T[G(x)] \\
& \text { s.t. } x \in X .
\end{aligned}
$$

Proof. Since $(\bar{x}, \bar{y})$ is a strictly efficient minimizer of (VP), by Theorem 14, there exists $\varphi \in C^{+i}$ such that

$$
\varphi[F(x)-\bar{y}] \geq 0, \quad \forall x \in A .
$$

Define $H: X \rightarrow 2^{\mathbb{R} \times Z}$ by

$$
H(x)=\varphi[F(x)-\bar{y}] \times G(x)=(\varphi F, G)(x)-\left(\varphi\left(\bar{y}, 0_{Z}\right)\right) .
$$

Since $(F, G)$ is nearly $C \times D$-convexlike on $X$, by Lemma 4 , we have that $H$ is nearly $\mathbb{R}_{+} \times D$-convexlike on $X$, while (27) implies that

$$
H(x) \cap\left[-\operatorname{int}\left(\mathbb{R}_{+} \times D\right)\right] \neq \emptyset, \quad \forall x \in X,
$$

has no solution, and hence, by Lemma 3 , there exists $(\lambda, \psi) \epsilon$ $\mathbb{R}_{+} \times D \backslash\left\{0_{Y^{*}}\right\}$ such that

$$
\lambda \varphi[F(x)-\bar{y}]+\psi[G(x)] \geq 0, \quad \forall x \in X .
$$

Since $\bar{x} \in A$, that is, $G(\bar{x}) \cap(-D) \neq \emptyset$, this implies that there exists $\bar{z} \in G(\bar{x})$ such that $-\bar{z} \in D$. Then, since $\psi \in D^{+}$, we get

$$
\psi(\bar{z}) \leq 0 .
$$

Also, let $x=\bar{x}$ in (30) and noting that $\bar{y} \in F(\bar{x})$, and $\bar{z} \in G(\bar{x})$, we get $\psi(\bar{z}) \geq 0$. 
Hence,

$$
\psi[G(\bar{x}) \cap-D]=0_{Y} .
$$

We now claim that $\lambda \neq 0$. If this is not the case, then

$$
\psi \in D^{+} \backslash\left\{0_{Y^{*}}\right\}
$$

By the generalized Slater constraint qualification, there exists $\tilde{x} \in X$ such that

$$
G(\tilde{x}) \cap-\operatorname{int} D \neq \emptyset,
$$

and so there exists $\widetilde{z} \in G(\tilde{x})$ such that

$$
-\widetilde{z} \in \operatorname{int} D \text {. }
$$

Hence, $\psi(\widetilde{z})<0$. But substituting $\lambda=0$ into (30), and by taking $x=\tilde{x}$ and $\widetilde{z} \in G(\tilde{x})$ in (30), we have

$$
\psi(\widetilde{z}) \geq 0
$$

This contradiction shows that $\lambda>0$. From this and $\varphi \in C^{+i}$, we can choose $c \in C \backslash\left\{0_{Y}\right\}$ such that $\lambda \varphi(c)=1$ and define the operator $T: Z \rightarrow Y$ by

$$
T(z)=\psi(z) c .
$$

Obviously,

$$
T \in L_{+}(Z, Y), \quad T[G(\bar{x}) \cap-D]=\left\{0_{Y}\right\} .
$$

Thus,

$$
0_{Y} \in T[G(\bar{x})], \quad \bar{y} \in F(\bar{x}) \subset F(\bar{x})+T[G(\bar{x})] .
$$

From (30) and (37), we obtain

$$
\begin{aligned}
\lambda \varphi[F & (x)+T G(x)] \\
& =\lambda \varphi[F(x)]+\psi[G(x)] \lambda \varphi(c) \\
& =\lambda \varphi[F(x)]+\psi[G(x)] \\
& \geq \lambda \varphi(\bar{y}), \quad \forall x \in X .
\end{aligned}
$$

Dividing the above inequality by $\lambda>0$, we obtain

$$
\varphi[F(x)+T G(x)] \geq \varphi(\bar{y}), \quad \forall x \in X .
$$

Since, $(F, G)$ is nearly $C \times D$-convexlike on $X$, by Lemma 4 , $F+T G$ is nearly $C$-convexlike on $X$. Therefore, by Theorem 13 and $\varphi \in C^{+i}$, we have that $(\bar{x} \cdot \bar{y})$ is a strictly efficient minimizer of (UVP).

Theorem 18. Let $\bar{x}, \bar{y} \in F(\bar{x})$ and let $C$ have a compact base. If there exists $T \in L_{+}(Z, Y)$ such that $0_{Y} \in T[G(\bar{x})]$ and $(\bar{x}, \bar{y})$ is a strictly efficient minimizer of (UVP), then $(\bar{x}, \bar{y})$ is a strictly efficient minimizer of $(V P)$.

Proof. From the assumption, we have

$$
\begin{gathered}
\bar{y} \in F(\bar{x}) \subset F(\bar{x})+T[G(\bar{x})], \\
(-C) \cap \mathrm{cl}\left[\bigcup_{x \in X}(F(x)+T[G(x)])+C-\bar{y}\right]=\left\{0_{Y}\right\} .
\end{gathered}
$$

Since $x \in A$, we have $G(x) \cap(-D) \neq \emptyset$. Thus there exists $z_{x} \in$ $G(x) \cap(-D)$. Then,

$$
-T\left(z_{x}\right) \in C
$$

which implies that $C-T\left(z_{x}\right) \subset C$; that is, $C \subset C+T\left(z_{x}\right)$, $\forall x \in A$. So, we have

$$
\begin{aligned}
& F(A)+C-\bar{y} \subset \bigcup_{x \in A}[F(x)+C-\bar{y}] \\
& \subset \bigcup_{x \in A}(F(x)+T[G(x)]+C-\bar{y}) \\
& \subset \bigcup_{x \in A}(F(x)+T[G(x)])+C-\bar{y} .
\end{aligned}
$$

This together with (43) implies

$$
(-C) \cap \operatorname{cl}[F(A)+C-\bar{y}]=\left\{0_{Y}\right\} .
$$

Noting that $\bar{x} \in A, \bar{y} \in F(\bar{x}) \subset F(A)$ and by Lemma 12, we have that $(\bar{x}, \bar{y})$ is a strictly efficient minimizer of (VP).

\section{Strict Efficiency and Duality}

Definition 19. The Lagrange map for (VP) is the set-valued map $\mathscr{L}: X \times L_{+}(Z, Y) \rightarrow 2^{Y}$ defined by

$$
\mathscr{L}(x, T)=F(x)+T[G(x)] .
$$

We denote

$$
\begin{aligned}
\mathscr{L}(X, T)=\bigcup_{x \in X} \mathscr{L}(x, T)=\bigcup_{x \in X}(F+T G)(x), \\
\mathscr{L}\left(x, L_{+}(Z, Y)\right)=\bigcup_{T \in L_{+}(Z, Y)} \mathscr{L}(x, T) \\
=\bigcup_{T \in L_{+}(Z, Y)}(F(x)+T[G(x)]) .
\end{aligned}
$$

Definition 20. The set-valued map $\Phi: L_{+}(Z, Y) \rightarrow 2^{Y}$ is defined as

$$
\Phi(T)=\operatorname{StE}[\mathscr{L}(X, T), C], \quad \forall T \in L_{+}(Z, Y),
$$

which is called a strict dual map of (VP).

Using this definition, we define the Lagrange dual problem associated with the primal problem (VP) as follows:

$$
\begin{aligned}
& C-\max \Phi(T) \\
& \text { s.t. } T \in L_{+}(Z, Y) \text {. }
\end{aligned}
$$

Definition 21. $\bar{y} \in \bigcup_{T \in L_{+}(Z, Y)} \Phi(T)$ is called an efficient point of (VD) if

$$
y-\bar{y} \notin C \backslash\left\{0_{Y}\right\}, \quad y \in \bigcup_{T \in L_{+}(Z, Y)} \Phi(T) .
$$

We can now establish the following dual theorems. 
Theorem 22 (weak duality). If $\bar{x} \in A$ and $y_{0} \in$ $\bigcup_{T \in L_{+}(Z, Y)} \Phi(T)$, then

$$
\left[y_{0}-F(\bar{x})\right] \cap\left(C \backslash\left\{0_{Y}\right\}\right)=\emptyset \text {. }
$$

Proof. From $y_{0} \in \bigcup_{T \in L_{+}(Z, Y)} \Phi(T)$, there exists $\bar{T} \in L_{+}(Z, Y)$ such that

$$
\begin{aligned}
y_{0} & \in \Phi(\bar{T}) \\
& \in \operatorname{StE}\left(\bigcup_{x \in X}[F(x)+\bar{T} G(x)], C\right) \\
& \subset E\left(\bigcup_{x \in X}[F(x)+\bar{T} G(x)], C\right) .
\end{aligned}
$$

Hence,

$$
\left(y_{0}-F(x)-\bar{T}[G(x)]\right) \cap\left(C \backslash\left\{0_{Y}\right\}\right)=\emptyset, \quad \forall x \in X .
$$

In particular,

$$
y_{0}-y-\bar{T}(z) \notin C \backslash\left\{0_{Y}\right\}, \quad \forall y \in F(\bar{x}), \forall z \in G(\bar{x}) .
$$

Noting that $\bar{x} \in A$, we choose $\bar{z} \in G(\bar{x}) \cap(-D)$. Then, $-\bar{T}(\bar{z}) \in$ $C$, and taking $z=\bar{z}$ in (54), we have

$$
y_{0}-y-\bar{T}(\bar{z}) \notin C \backslash\left\{0_{Y}\right\}, \quad \forall y \in F(\bar{x}) .
$$

Hence, from $-\bar{T}(\bar{z}) \in C$ and $C+C \backslash\left\{0_{Y}\right\} \subseteq C \backslash\left\{0_{Y}\right\}$, we obtain

$$
y_{0}-y \notin C \backslash\left\{0_{Y}\right\}, \quad \forall y \in F(\bar{x}) .
$$

This completes the proof.

Theorem 23 (strong duality). Let $F$ be nearly $C$-convexlike on $X$. Let $(F, G)$ be nearly $(C \times D)$-convexlike on $X$ and let $C$ have a compact base. Furthermore, let (VP) satisfy the generalized Slater constraint qualification. If $\bar{x}$ is a strictly efficient solution of $(V P)$, then there exists that $\bar{y} \in F(\bar{x})$ is an efficient point of $(V D)$.

Proof. Since $\bar{x}$ is a strictly efficient solution of (VP), then there exists $\bar{y} \in F(\bar{x})$ such that $(\bar{x}, \bar{y})$ is a strictly efficient minimizer of (VP). According to Theorem 17, there exists $T \in L_{+}(Z, Y)$ such that $T[G(\bar{x}) \cap(-D)]=\left\{0_{Y}\right\}$, and $(\bar{x}, \bar{y})$ is a strictly efficient minimizer of (UVP). Hence, $\bar{y} \in \operatorname{StE}\left[\bigcup_{x \in X}(F(x)+\right.$ $T[G(x)]), C]=\Phi(T) \subset \bigcup_{T \in L_{+}(Z, Y)} \Phi(T)$. By Theorem 22, we have

$$
(y-\bar{y}) \notin C \backslash\left\{0_{Y}\right\}, \quad \forall y \in \bigcup_{T \in L_{+}(Z, Y)} \Phi(T) .
$$

Therefore, by Definition 20, we know that $\bar{y}$ is an efficient point of (VD).

\section{Strict Efficient and Strict Saddle Point}

We will now introduce a new concept of strict saddle point for a set-valued Lagrange map $\mathscr{L}$ and use it to characterize strict efficiency.

For a nonempty subset $S$ of $Y$, we define a set

$$
\begin{aligned}
\operatorname{StM}(S, C)=\{\bar{y} \in S \mid \forall \epsilon>0, \exists \delta>0 \\
\text { such that }(S-\bar{y}) \cap(-\delta B+C) \subseteq \epsilon B\} .
\end{aligned}
$$

It is easy to find that $\bar{y} \in \operatorname{StM}(S, C)$ if and only if $-\bar{y} \in$ $\operatorname{StE}(-S, C)$, and if $Y$ is normed space and $C$ has a compact base. Then by Lemma 12, we have $\bar{y} \in \operatorname{StM}(S, C)$ if and only if $\mathrm{cl}(S-C-\bar{y}) \cap C=\left\{0_{Y}\right\}$.

Definition 24. A pair $(\bar{x}, \bar{T}) \in X \times L_{+}(Z, Y)$ is said to be a strict saddle point of Lagrange map $\mathscr{L}$ if

$$
\begin{aligned}
\mathscr{L}(\bar{x}, \bar{T}) & \cap \operatorname{StE}\left[\bigcup_{x \in X} \mathscr{L}(x, \bar{T}), C\right] \\
& \cap \operatorname{StM}\left[\bigcup_{T \in L_{+}(Z, Y)} \mathscr{L}(\bar{x}, T), C\right] \neq \emptyset .
\end{aligned}
$$

We first present an important equivalent characterization for a strict saddle point of the Lagrange map $\mathscr{L}$.

Lemma 25. Let $C$ have a compact base. $(\bar{x}, \bar{T}) \in X \times L_{+}(Z, Y)$ is said to be a strict saddle point of Lagrange map $\mathscr{L}$ if only if there exist $\bar{y} \in F(\bar{x})$ and $\bar{z} \in G(\bar{x})$ such that

$$
\begin{aligned}
& \text { (i) } \bar{y} \quad \in \quad \operatorname{StE}\left[\bigcup_{x \in X} \mathscr{L}(x, \bar{T}), C\right] \\
& \operatorname{StM}\left[\bigcup_{T \in L_{+}(Z, Y)} \mathscr{L}(\bar{x}, T), C\right] \text {, }
\end{aligned}
$$

(ii) $\bar{T}(\bar{z})=0_{Y}$.

Proof (necessity). Since $(\bar{x}, \bar{T})$ is a strict saddle point of the map $\mathscr{L}$, by Definition 24 there exists $\bar{y} \in F(\bar{x}), \bar{z} \in G(\bar{x})$ such that

$$
\begin{gathered}
\bar{y}+\bar{T}(\bar{z}) \in \operatorname{StE}\left[\bigcup_{x \in X} \mathscr{L}(x, \bar{T}), C\right], \\
\bar{y}+\bar{T}(\bar{z}) \in \operatorname{StM}\left[\bigcup_{T \in L_{+}(Z, Y)} \mathscr{L}(\bar{x}, T), C\right] .
\end{gathered}
$$

From (61), we have

$$
C \cap \mathrm{cl}\left[\bigcup_{T \in L_{+}(Z, Y)} \mathscr{L}(\bar{x}, T)-C-(\bar{y}+\bar{T}(\bar{z}))\right]=\left\{0_{Y}\right\}
$$


Since every $T \in L_{+}(Z, Y)$, we have

$$
\begin{aligned}
T(\bar{z})- & \bar{T}(\bar{z}) \\
= & {[\bar{y}+T(\bar{z})]-[\bar{y}+\bar{T}(\bar{z})] } \\
& \in F(\bar{x})+T[G(\bar{x})]-[\bar{y}+\bar{T}(\bar{z})] \\
= & \mathscr{L}(\bar{x}, T)-[\bar{y}+\bar{T}(\bar{z})] .
\end{aligned}
$$

We have

$$
\begin{aligned}
& \left\{T(\bar{z}): T \in L_{+}(Z, Y)\right\}-C-\bar{T}(\bar{z}) \\
& \quad \subset \bigcup_{T \in L_{+}(Z, Y)} \mathscr{L}(\bar{x}, T)-C-[\bar{y}+\bar{T}(\bar{z})] .
\end{aligned}
$$

Hence,

$$
\begin{aligned}
& \mathrm{cl}\left[\bigcup_{T \in L_{+}(Z, Y)}\{T(\bar{z})\}-C-\bar{T}(\bar{z})\right] \\
& \quad \mathrm{c} \mathrm{cl}\left[\bigcup_{T \in L_{+}(Z, Y)} \mathscr{L}(\bar{x}, T)-C-[\bar{y}+\bar{T}(\bar{z})]\right] .
\end{aligned}
$$

Thus, from (62), we have

$$
C \cap \mathrm{cl}\left[\bigcup_{T \in L_{+}(Z, Y)}\{T(\bar{z})\}-C-\bar{T}(\bar{z})\right]=\left\{0_{Y}\right\} .
$$

Let $f: L(Z, Y) \rightarrow Y$ be defined by

$$
f(T)=-T(\bar{z}) .
$$

Then, (66) can be written as

$$
(-C) \cap \mathrm{cl}\left[f\left(L_{+}(Z, Y)\right)+C-f(\bar{T})\right]=\left\{0_{Y}\right\} .
$$

This together with Lemma 12 shows that $\bar{T} \in L_{+}(Z, Y)$ is a strictly efficient point of the vector optimization problem

$$
\begin{aligned}
& C-\min f(T) \\
& \text { s.t. } T \in L_{+}(Z, Y) .
\end{aligned}
$$

Since $f$ is a linear map, then of course $-f$ is nearly $C$ convexlike on $L_{+}(Z, Y)$. Hence, by Theorem 14, there exists $\varphi \in C^{+i}$ such that

$$
\begin{aligned}
\varphi[-\bar{T}(\bar{z})] & =\varphi[f(\bar{T})] \leq \varphi[f(T)] \\
& =\varphi[-T(\bar{z})], \quad \forall T \in L_{+}(Z, Y) .
\end{aligned}
$$

Now, we claim that

$$
-\bar{z} \in D
$$

If this is not true, then since $D$ is a closed convex cone set, by the strong separation theorem in topological vector space [21], there exists $\mu \in Z^{*} \backslash\left\{0_{Z^{*}}\right\}$ such that

$$
\mu(-\bar{z})<\mu(\lambda d), \quad \forall d \in D, \forall \lambda>0 .
$$

In the above expression, taking $d=0_{z} \in D$ gets

$$
\mu(\bar{z})>0
$$

while letting $\lambda \rightarrow+\infty$ leads to

$$
\mu(d) \geq 0, \quad \forall d \in D .
$$

Hence,

$$
\mu \in D^{+} \backslash\left\{0_{Z^{*}}\right\} .
$$

Let $c^{*} \in \operatorname{int} C$ be fixed, and define $T^{*}: Z \rightarrow Y$ as

$$
T^{*}(z)=\left[\frac{\mu(z)}{\mu(\bar{z})}\right] c^{*}+\bar{T}(z) .
$$

It is evident that $T^{*} \in L(Z, Y)$ and that

$$
T^{*}(d)=\left[\frac{\mu(d)}{\mu\left(\bar{z} c^{*}\right)}\right]+\bar{T}(d) \in C+C \subset C, \quad \forall d \in D .
$$

Hence, $T^{*} \in L_{+}(Z, Y)$. Taking $z=\bar{z}$ in (66), we obtain

$$
T^{*}(\bar{z})-\bar{T}(\bar{z})=c^{*} .
$$

Hence,

$$
\varphi\left[T^{*}(\bar{z})\right]-\varphi[\bar{T}(\bar{z})]=\varphi\left(c^{*}\right)>0
$$

which contradicts (70). Therefore,

$$
-\bar{z} \in D
$$

Thus, $-\bar{T}(\bar{z}) \in C$, since $\bar{T} \in L_{+}(Z, Y)$. If $\bar{T}(\bar{z}) \neq 0_{Y}$, then

$$
-\bar{T}(\bar{z}) \in C \backslash\left\{0_{Y}\right\}
$$

hence, $\varphi[\bar{T}(\bar{z})]<0$, by $\varphi \in C^{+i}$, while taking $T=0 \in$ $L_{+}(Z, Y)$ leads to

$$
\varphi(\bar{T}(\bar{z})) \geq 0
$$

This contradiction shows that $\bar{T}(\bar{z})=0_{Y}$, that is, condition (ii) holds.

Therefore, by (60) and (61), we know

$$
\begin{aligned}
& \bar{y} \in \operatorname{StE}\left[\bigcup_{x \in X} \mathscr{L}(x, \bar{T}), C\right] \\
& \cap \operatorname{StM}\left[\bigcup_{T \in L_{+}(Z, Y)} \mathscr{L}(\bar{x}, T), C\right] ;
\end{aligned}
$$

that is, condition (i) holds.

Sufficiency. From $\bar{y} \in F(\bar{x}), \bar{z} \in G(\bar{x})$, and condition (ii), we get

$$
\bar{y}=\bar{y}+\bar{T}(\bar{z}) \in F(\bar{x})+\bar{T}[G(\bar{x})]=\mathscr{L}(\bar{x}, \bar{T}) .
$$


And by condition (i), we obtain

$$
\begin{gathered}
\bar{y} \in \mathscr{L}(\bar{x}, \bar{T}) \cap \operatorname{StE}\left[\bigcup_{x \in X} \mathscr{L}(x, \bar{T}), C\right] \\
\cap \operatorname{StM}\left[\bigcup_{T \in L_{+}(Z, Y)} \mathscr{L}(\bar{x}, T), C\right] .
\end{gathered}
$$

Therefore, $(\bar{x}, \bar{T})$ is a strict saddle point of the set-valued Lagrange map $\mathscr{L}$, and the proof is complete.

The following saddle-point theorems allow us to express a strictly efficient solution of (VP) as a strict saddle of the setvalued Lagrange map $\mathscr{L}$.

Theorem 26. Let $F$ be nearly $C$-convexlike on $A$, let $(F, G)$ be nearly $(C \times D)$-convexlike on $A$, and let $C$ have a compact base. Moreover, (VP) satisfies generalized Slater constrained qualification.

(i) If $(\bar{x}, \bar{T})$ is a strict saddle point of the map $\mathscr{L}$, then $\bar{x}$ is strictly efficient solution of $(V P)$.

(ii) If $(\bar{x}, \bar{y})$ is a strictly efficient minimizer of $(V P)$, and $\bar{y} \in \operatorname{StM}\left[\bigcup_{T \in L_{+}(Z, Y)} \mathscr{L}(\bar{x}, T), C\right]$, then there exists $\bar{T} \epsilon$ $L_{+}(Z, Y)$ such that $(\bar{x}, \bar{T})$ is a strict saddle point of the map $\mathscr{L}$.

Proof. (i) By the necessity of Lemma 25, we have

$$
0_{Y} \in \bar{T}[G(\bar{x})],
$$

and there exists $\bar{y} \in F(\bar{x})$ such that $(\bar{x}, \bar{y})$ is a strictly efficient minimizer of the problem

$$
\begin{aligned}
& C-\min F(x)+\bar{T}[G(x)] \\
& \text { s.t. } x \in X .
\end{aligned}
$$

According to Theorem $18,(\bar{x}, \bar{y})$ is a strictly efficient minimizer of (VP). Therefore, $\bar{x}$ is strictly efficient solution of (VP).

(ii) From the assumption, and by the Theorem 17, there exists $\bar{T} \in L_{+}(Z, Y)$ such that

$$
\begin{gathered}
\bar{y} \in \operatorname{StE}\left[\bigcup_{x \in X} \mathscr{L}(x, \bar{T}), C\right], \\
T[G(\bar{x}) \cap(-D)]=0_{Y} .
\end{gathered}
$$

Therefore, there exists $\bar{z} \in G(\bar{x})$ such that $\bar{T}(\bar{z})=0_{Y}$. Hence, from Lemma 25 , it follows that $(\bar{x}, \bar{T})$ is a strict saddle point of the map $\mathscr{L}$.

Lemma 27. Let $(\bar{x}, \bar{T}) \in X \times L_{+}(Z, Y), \bar{y} \in F(\bar{x})$, and $\bar{z} \in$ $G(\bar{x})$. Then the following conditions:

$$
\begin{aligned}
& \text { (a) } \bar{y} \quad \in \quad \operatorname{StE}\left[\bigcup_{x \in X} \mathscr{L}(x, \bar{T}), C\right] \\
& \operatorname{StM}\left[\bigcup_{T \in L_{+}(Z, Y)} \mathscr{L}(\bar{x}, T), C\right] \text {, }
\end{aligned}
$$

(b) $\bar{T}(\bar{z})=0_{Y}$,

are equivalent to the following conditions:

$$
\begin{aligned}
& \text { (i) } \bar{y} \in \operatorname{StE}\left[\bigcup_{x \in X} \mathscr{L}(x, \bar{T}), C\right] \cap \operatorname{StM}[F(\bar{x}), C], \\
& \text { (ii) } G(\bar{x}) \subset-D \\
& \text { (iii) } \bar{T}(\bar{z})=0_{Y} .
\end{aligned}
$$

Proof. By conditions (a) and (b), it is easy to verify that conditions (i) and (iii) hold. Now, we show that $G(\bar{x}) \subset-D$. If this is not true, then there would exist $z_{0} \in G(\bar{x})$ such that

$$
-z_{0} \notin D
$$

Then, since $D$ is a closed convex set, by the strong separation theorem in topological vector space (see [21]), there exists $\psi_{0} \in Z^{*} \backslash\left\{0_{Z^{*}}\right\}$ such that

$$
\psi_{0}\left(-z_{0}\right)<\psi(\lambda d), \quad \forall d \in D, \forall \lambda>0 .
$$

In the above expression, taking $d=0_{z} \in D$ gives

$$
\psi_{0}\left(z_{0}\right)>0
$$

and taking $\lambda \rightarrow+\infty$ leads to

$$
\psi_{0}(d) \geq 0, \quad \forall d \in D .
$$

Hence,

$$
\psi_{0} \in D^{+} \backslash\left\{0_{z^{*}}\right\} .
$$

Take $c_{0} \in$ int $C$ and define $T_{0}: Z \rightarrow Y$ as

$$
T_{0}(z)=\psi_{0}(z) c_{0} .
$$

Then, $T_{0} \in \mathfrak{Ł}_{+}(Z, Y)$ and

$$
T_{0}\left(z_{0}\right)=\psi_{0}\left(z_{0}\right) c_{0} \in \operatorname{int} C \subset C \backslash\left\{0_{Y}\right\} .
$$

And, by condition (a), we have

$$
y-\bar{y} \notin C \backslash\left\{0_{Y}\right\}, \quad \forall y \in \bigcup_{T \in L_{+}(Z, Y)} \mathscr{L}(\bar{x}, T) .
$$

From

$$
\begin{aligned}
\bar{y}+T_{0}\left(z_{0}\right) \in F(\bar{x})+T_{0}[G(\bar{x})] \\
=L\left(\bar{x}, T_{0}\right) \subset \bigcup_{T \in L_{+}(Z, Y)} \mathscr{L}(\bar{x}, T),
\end{aligned}
$$

and by (95), we have

$$
T_{0}\left(z_{0}\right)=\left[\bar{y}+T_{0}\left(z_{0}\right)\right]-\bar{y} \notin C \backslash\left\{0_{Y}\right\} .
$$

This conflicts with (99). Therefore, $G(\bar{x}) \subset-D$.

Conversely, by (i), we have

$$
\bar{y} \in \operatorname{StM}[F(\bar{x}), C],
$$

that is,

$$
\operatorname{cl}[F(\bar{x})-C-\bar{y}] \cap C=\left\{0_{Y}\right\} .
$$


By condition (ii), for every $T \in L_{+}(Z, Y)$, we have

$$
T[G(\bar{x})] \subset T(-D) \subset-C .
$$

Thus,

$$
\begin{aligned}
\bigcup_{x \in X} \mathscr{L} & (\bar{x}, T)-C-\bar{y} \\
& =\bigcup_{x \in X}[F(\bar{x})+T[G(\bar{x})]]-C-\bar{y} \\
& =\bigcup_{x \in X} T[G(\bar{x})]+F(\bar{x})-C-\bar{y} \\
& \subset F(\bar{x})-C-C-\bar{y} \\
& \subset F(\bar{x})-C-\bar{y} .
\end{aligned}
$$

Therefore, by (99), we have

$$
\mathrm{cl}\left[\bigcup_{x \in X} \mathscr{L}(\bar{x}, T)-C-\bar{y}\right] \cap C=\left\{0_{Y}\right\},
$$

that is, $\bar{y} \in \operatorname{StM}\left[\bigcup_{x \in X} \mathscr{L}(\bar{x}, T)\right]$. Therefore, this proof is completed.

By Lemmas 25 and 27 and Theorem 26, we can obtain immediately the following corollary.

Corollary 28. Let $F$ be nearly $C$-convexlike on $A$, let $(F, G)$ be nearly $(C \times D)$-convexlike on $A$, and let $(V P)$ satisfy generalized Slater constrained qualification.

(i) If $(\bar{x}, \bar{T})$ is a strict saddle point of the map $\mathscr{L}$, then $\bar{x}$ is strictly efficient solution of $(V P)$.

(ii) If $(\bar{x}, \bar{y})$ is a strictly efficient minimizer of $(V P)$ and $G(\bar{x}) \subset-D, \bar{y} \in \operatorname{StM}[F(\bar{x}), C]$, then there exists $\bar{T} \in L_{+}(Z, Y)$ such that $(\bar{x}, \bar{T})$ is a strict saddle point of the map $\mathscr{L}$.

\section{Acknowledgments}

Zhimiao Fang's research was supported by the Natural Science Foundation Project of CQ CSTC (Grant no. cstc2012jjA00033). The authors would like to thank two anonymous referees for their valuable comments and suggestions, which helped to improve the paper.

\section{References}

[1] H. W. Kuhn and A. W. Tucker, "Nonlinear programming," in Proceedings of the 2nd Berkeley Symposium on Mathematical Statistics and Probability, pp. 481-492, University of California Press, Los Angeles, Calif, USA, 1951.

[2] A. M. Geoffrion, "Proper efficiency and the theory of vector maximization," Journal of Mathematical Analysis and Applications, vol. 22, pp. 618-630, 1968.

[3] J. Borwein, "Proper efficient points for maximizations with respect to cones," SIAM Journal on Control and Optimization, vol. 15, no. 1, pp. 57-63, 1977.
[4] R. Hartley, "On cone-efficiency, cone-convexity and cone-compactness," SIAM Journal on Applied Mathematics, vol. 34, no. 2, pp. 211-222, 1978.

[5] H. P. Benson, "An improved definition of proper efficiency for vector maximization with respect to cones," Journal of Mathematical Analysis and Applications, vol. 71, no. 1, pp. 232-241, 1979.

[6] M. I. Henig, "Proper efficiency with respect to cones," Journal of Optimization Theory and Applications, vol. 36, no. 3, pp. 387407, 1982.

[7] J. M. Borwein and D. Zhuang, "Super efficiency in vector optimization," Transactions of the American Mathematical Society, vol. 338, no. 1, pp. 105-122, 1993.

[8] E. Bednarczuk and W. Song, "PC points and their application to vector optimization," Pliska Studia Mathematica Bulgarica, vol. 12, pp. 21-30, 1998.

[9] A. Zaffaroni, "Degrees of efficiency and degrees of minimality," SIAM Journal on Control and Optimization, vol. 42, no. 3, pp. 1071-1086, 2003.

[10] H. W. Corley, "Existence and Lagrangian duality for maximizations of set-valued functions," Journal of Optimization Theory and Applications, vol. 54, no. 3, pp. 489-500, 1987.

[11] Z.-F. Li and G.-Y. Chen, "Lagrangian multipliers, saddle points, and duality in vector optimization of set-valued maps," Journal of Mathematical Analysis and Applications, vol. 215, no. 2, pp. 297-316, 1997.

[12] W. Song, "Lagrangian duality for minimization of nonconvex multifunctions," Journal of Optimization Theory and Applications, vol. 93, no. 1, pp. 167-182, 1997.

[13] G. Y. Chen and J. Jahn, "Optimality conditions for set-valued optimization problems," Mathematical Methods of Operations Research, vol. 48, no. 2, pp. 187-200, 1998.

[14] W. D. Rong and Y. N. Wu, "Characterizations of super efficiency in cone-convexlike vector optimization with set-valued maps," Mathematical Methods of Operations Research, vol. 48, no. 2, pp. 247-258, 1998.

[15] X. M. Yang, D. Li, and S. Y. Wang, "Near-subconvexlikeness in vector optimization with set-valued functions," Journal of Optimization Theory and Applications, vol. 110, no. 2, pp. 413-427, 2001.

[16] S. J. Li, X. Q. Yang, and G. Y. Chen, "Nonconvex vector optimization of set-valued mappings," Journal of Mathematical Analysis and Applications, vol. 283, no. 2, pp. 337-350, 2003.

[17] Z. F. Li, "Benson proper efficiency in the vector optimization of set-valued maps," Journal of Optimization Theory and Applications, vol. 98, no. 3, pp. 623-649, 1998.

[18] A. Mehra, "Super efficiency in vector optimization with nearly convexlike set-valued maps," Journal of Mathematical Analysis and Applications, vol. 276, no. 2, pp. 815-832, 2002.

[19] L. Y. Xia and J. H. Qiu, "Superefficiency in vector optimization with nearly subconvexlike set-valued maps," Journal of Optimization Theory and Applications, vol. 136, no. 1, pp. 125-137, 2008.

[20] E. Miglierina, "Characterization of solutions of multiobjective optimization problem," Rendiconti del Circolo Matematico di Palermo, vol. 50, no. 1, pp. 153-164, 2001.

[21] J. Jahn, Mathematical Vector Optimization in Partially Ordered Linear Spaces, vol. 31 of Methods and Procedures in Mathematical Physics, Peter D. Lang, Frankfurt, Germany, 1986.

[22] J.-B. Hiriart-Urruty, "Tangent cones, generalized gradients and mathematical programming in Banach spaces," Mathematics of Operations Research, vol. 4, no. 1, pp. 79-97, 1979. 


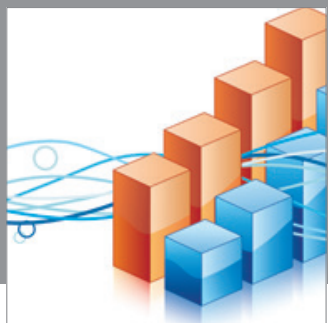

Advances in

Operations Research

mansans

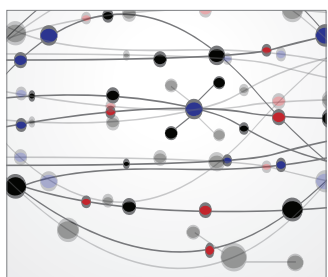

The Scientific World Journal
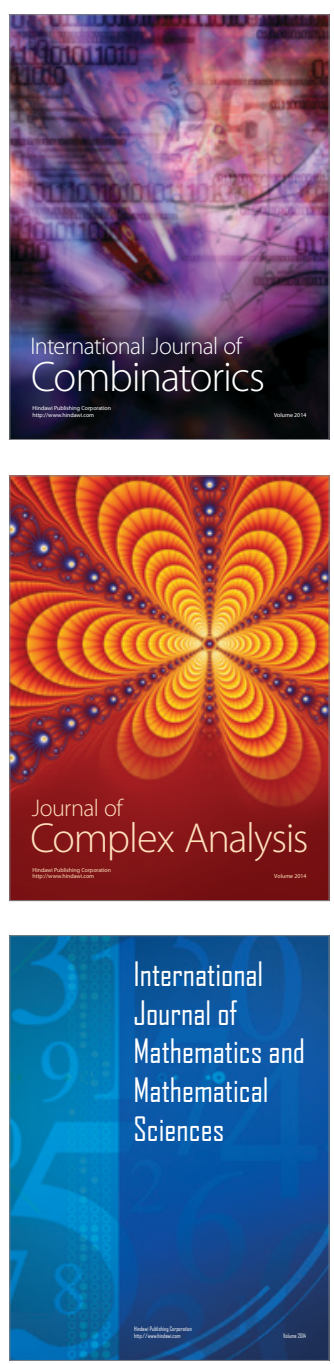
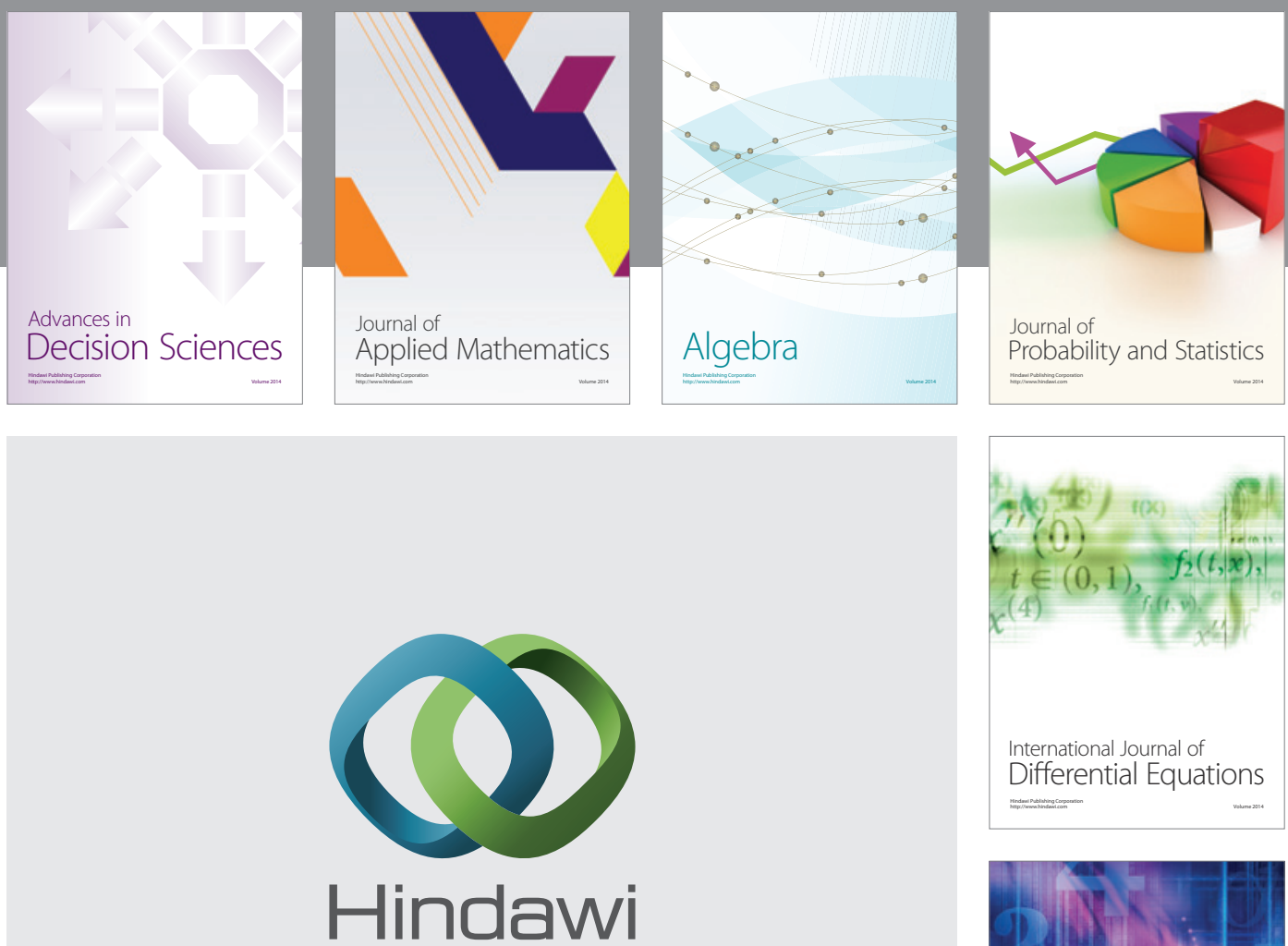

Submit your manuscripts at http://www.hindawi.com
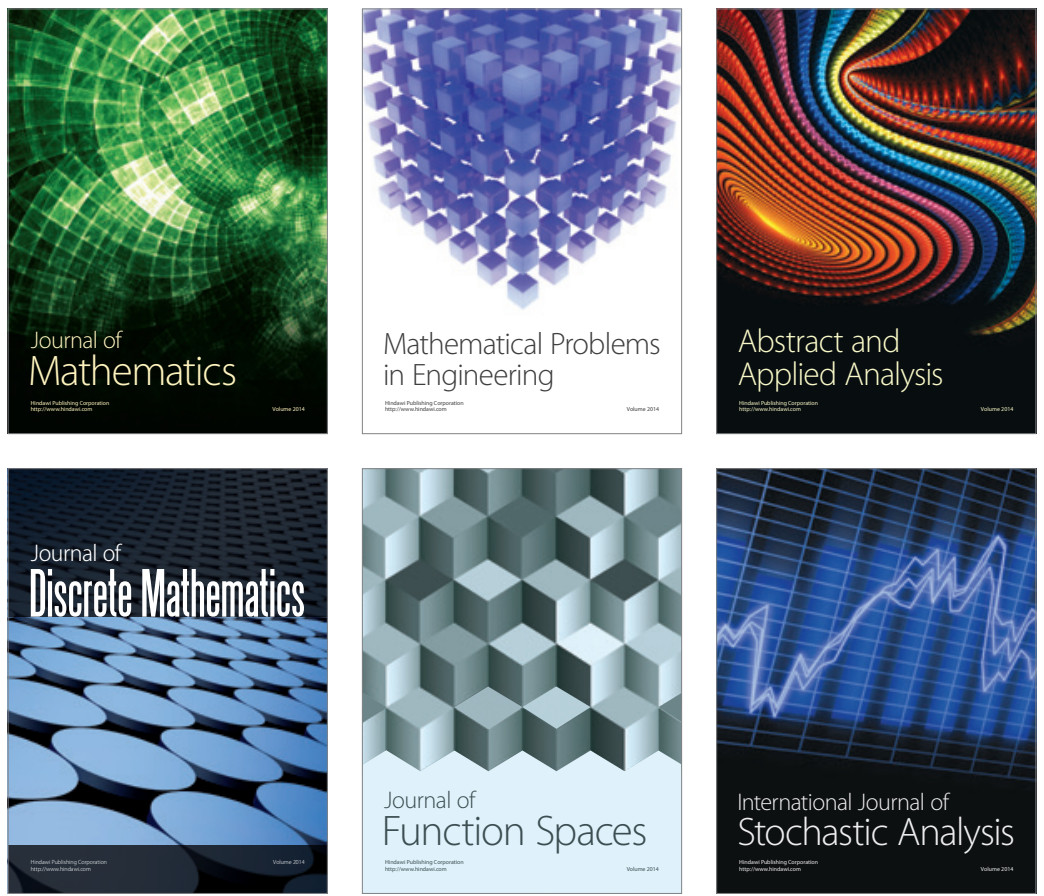

Journal of

Function Spaces

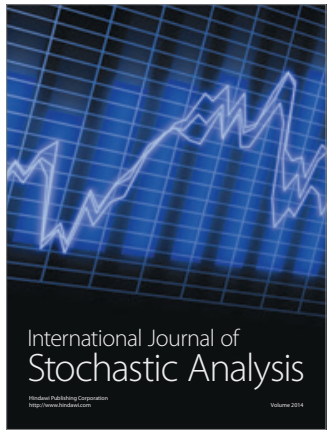

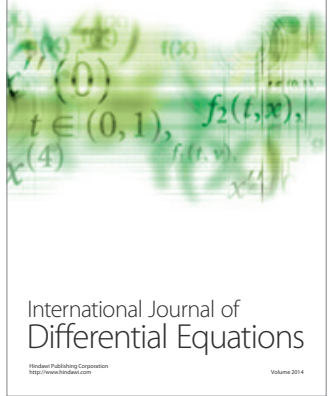
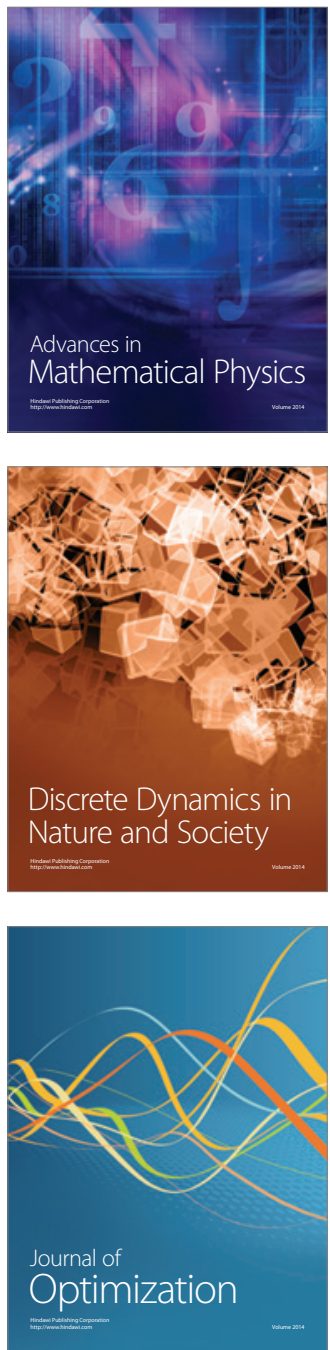